\title{
Why No Windows (Was: A Thin Window for the SubHarmonic Drive Experiment)
}

\author{
M. Hill and D. Walz
}




\title{
Why No Windows \\ (Was: A Thin Window for the SubHarmonic Drive Experiment)
}

\author{
Marc Hill Dieter Walz
}

March 24, 1998

\begin{abstract}
We discuss the window requirements for isolating the vacuum chamber for the subharmonic drive experiment from the NLCTA vacuum. We consider mechanical and heat stress, as well as looking at multiple scattering due to the foil. We decide that a window would not be appropriate because of multiple scattering.
\end{abstract}

\section{Introduction}

The worst case NLCTA beam parameters at the vacuum chamber would be:

- $340 \mathrm{MeV}$ electron beam energy

- 1400 bunches $\times 510^{8} \mathrm{e}^{-} /$bunch $\times 10 \mathrm{~Hz}$

- $100 \times 600 \mu \mathrm{m}^{2}$ beam area (worst case)

- normalized emittance of $50 \mathrm{~mm} \mathrm{mrad}$

Most values are calculated assuming the use of a $25 \mu \mathrm{m}$ thick stainless steel foil as a window.

\section{Heat Load}

The power dissipated in the window is

$$
P_{w}=\left(-\rho \frac{d E}{d x}\right) N e R \delta
$$


where $\rho$ is the density, $\frac{d E}{d x}$ the energy loss in the material, $N$ is the number of electrons, $e$ the electron charge, $R$ the repetition rate and $\delta$ is the thickness of the material.

For stainless steel, $\left(-\rho \frac{d E}{d x}\right)=11.7 \mathrm{MeV} / \mathrm{cm}$ and we will consider a $25 \mu \mathrm{m}$ thick foil to get

$$
P_{w}=3.3 \mathrm{~mW} \text {. }
$$

Assuming a guassian distribution $(100 \mu \mathrm{m} \times 600 \mu \mathrm{m})$, we get a pulsed temperature rise of

$$
\Delta T=\frac{P_{w}}{C_{h}}=70^{\circ} \mathrm{C}
$$

where $C_{h}$ includes the volume of material being heated. This pulsed heating is large, but not unreasonable for stainless steel.

\section{Hemi-spherical Stress}

Using a saftey factor of 2 on the atmospheric pressure, the mechanical stress is

$$
\sigma=\frac{\operatorname{Pr}}{2 \delta}=7,400 \mathrm{psi}
$$

where $P$ is the pressure differential, $r=0.5$ inches, the radius of curvature and $\delta=25 \mu \mathrm{m}$ is the foil thickness. The stress is well under the yeild strength for stainless, $\approx 30,000$ psi.

\section{Multiple Scattering}

With $\beta=5 \mathrm{~m}$, the angular spread of the beam at the window is

$$
<\sigma_{x}^{\prime}>=\sqrt{\frac{\epsilon_{n}}{\beta \gamma}} \approx 0.1 \mathrm{mrad}
$$

The multiple scattering due to a $25 \mu \mathrm{m}$ foil is [1]

$$
\begin{gathered}
\theta_{o}=\frac{13.6 \mathrm{MeV}}{\beta c p} \sqrt{\frac{\delta}{X_{o}}}\left[1+0.038 \ln \frac{\delta}{X_{o}}\right]=1.3 \mathrm{mrad} \\
\rho X_{o}=\frac{716.4 A}{Z(Z+1) \ln \left(\frac{287}{\sqrt{Z}}\right)} \frac{\mathrm{g}}{\mathrm{cm}^{2}}
\end{gathered}
$$

where $A$ is the atomic mass, $Z$ the atomic number and $\beta c p=300 \mathrm{MeV}$. For stainless steel $X_{o}=1.8 \mathrm{~cm}$. This foil would greatly increase the opening angle of the beam and hence destroy the beam emittance.

Plot 1 shows the scattering angle versus foil thickness for beryllium and stainless steel. From this plot, we can see that to reduce the multiple scattering to the level of the natural angular spread of the beam, we could take 


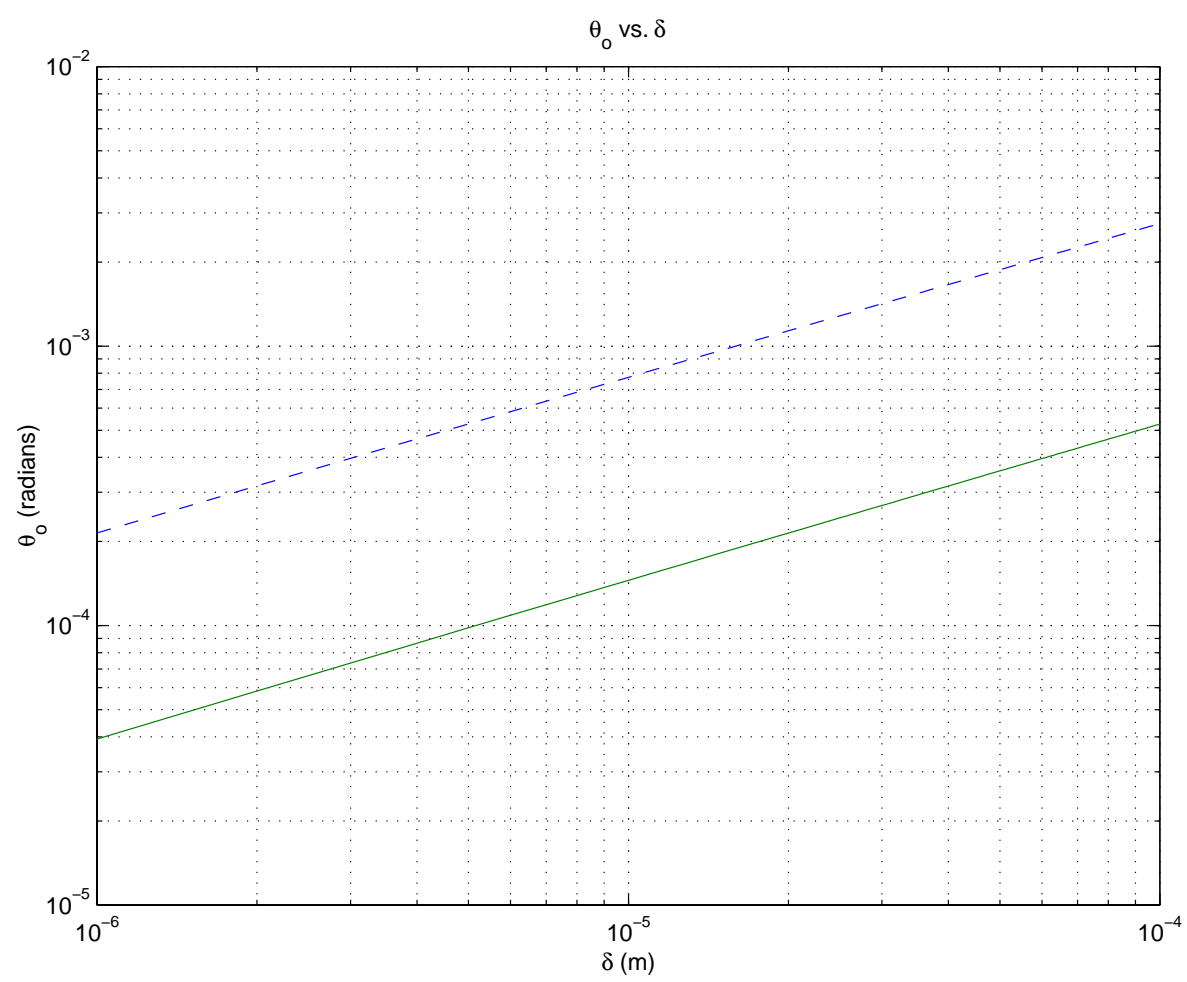

Figure 1: Multiple scattering for Stainless Steel (upper) and Beryllium (lower).

a maximum foil thickness of $7 \mu \mathrm{m}$ for beryllium and not possible for stainless steel. A beryllium window of this thickness would be too thin to consider practical for this experiment (i.e. not worth the trouble).

\section{References}

[1] Particle Data Group, Particle Data Book, Phys Rev D50 1173 (1994) 